ARTICLE

\title{
Embedding atomic cobalt into graphene lattices to activate room-temperature ferromagnetism
}

Wei Hu${ }^{1}$, Chao Wang ${ }^{1 凶}$, Hao Tan ${ }^{1}$, Hengli Duan ${ }^{1}$, Guinan $\mathrm{Li}^{1}, \mathrm{Na} \mathrm{Li}{ }^{1}$, Qianqian $\mathrm{Ji}^{1}$, Ying Lu${ }^{1}$, Yao Wang ${ }^{1}$, Zhihu Sun (1) ${ }^{1 凶}$, Fengchun $\mathrm{Hu}^{1} \&$ Wensheng Yan (1) ${ }^{1 凶}$

Graphene is extremely promising for next-generation spintronics applications; however, realizing graphene-based room-temperature magnets remains a great challenge. Here, we demonstrate that robust room-temperature ferromagnetism with $T_{\mathrm{C}}$ up to $\sim 400 \mathrm{~K}$ and saturation magnetization of $0.11 \mathrm{emu} \mathrm{g}^{-1}(300 \mathrm{~K})$ can be achieved in graphene by embedding isolated $\mathrm{Co}$ atoms with the aid of coordinated $\mathrm{N}$ atoms. Extensive structural characterizations show that square-planar $\mathrm{Co}-\mathrm{N}_{4}$ moieties were formed in the graphene lattices, where atomically dispersed $\mathrm{Co}$ atoms provide local magnetic moments. Detailed electronic structure calculations reveal that the hybridization between the $d$ electrons of Co atoms and delocalized $p_{z}$ electrons of $\mathrm{N} / \mathrm{C}$ atoms enhances the conduction-electron mediated long-range magnetic coupling. This work provides an effective means to induce room-temperature ferromagnetism in graphene and may open possibilities for developing graphene-based spintronics devices.

\footnotetext{
${ }^{1}$ National Synchrotron Radiation Laboratory, University of Science and Technology of China, Hefei, P. R. China. ${ }^{凶}$ email: chaowng@ustc.edu.cn; zhsun@ustc.edu.cn; ywsh2000@ustc.edu.cn
} 
G raphene is a two-dimensional (2D) layered material composed of a one-atom-thick hexagonal network of $s p^{2}$ hybridized carbon ${ }^{1,2}$. It possesses many unique properties such as large specific surface area ${ }^{3}$, superior mechanical strength ${ }^{4}$, high intrinsic carrier mobility 5,6 , and thermal conductivity ${ }^{7}$. Great efforts have been devoted to promoting the applications of graphene in a diversity of fields including field-effect transistors ${ }^{8}$, chemical sensors ${ }^{9}$, fuel cells ${ }^{10}$, and so forth. Graphene is also envisaged to be a highly promising material for next-generation spintronic applications, owing to its extraordinary carrier mobility, long spin diffusion length, weak intrinsic spin-orbit coupling, and limited hyperfine interactions ${ }^{11,12}$. For practical applications in spintronic devices, the graphene-based material is required to be ferromagnetic above room temperature. Unfortunately, pristine graphene is intrinsically diamagnetic and lacks local magnetic moments ${ }^{13,14}$. Therefore, development of effective strategies to induce and manipulate the ferromagnetism of graphene has become a key issue to extend its applications in spintronics.

So far, various strategies have been attempted to realize ferromagnetic ordering in graphene, including utilization of atomic vacancies $^{15}, s p^{3}$ functionlization ${ }^{16}$, chemical doping ${ }^{14,17}$, surface adsorption ${ }^{18}$, and zigzag edges ${ }^{19}$. However, the magnetic moments induced by point defects or edges are relatively weak and unstable; and consequently the ferromagnetic ordering often collapses at rather low temperatures ${ }^{14,16,17,20,21}$. For instance, Grigorieva and colleagues ${ }^{21}$ produced high-density vacancy defects on graphene with ion beam radiation and realized a macroscopic magnetic moment of $0.001 \mu_{\mathrm{B}} /$ atom; but no ferromagnetic coupling was observed at temperatures as low as liquid helium (4.2 K). Tucek et al. ${ }^{14}$ found that S-doped graphene could only maintain ferromagnetic ordering at temperatures below 62 $\mathrm{K}$, and Liu et al. ${ }^{22}$ demonstrated that only pure diamagnetism can be observed in $\mathrm{N}$-doped graphene at $300 \mathrm{~K}$. To improve the stability of ferromagnetic coupling, the common wisdom is to increase the content of defects so as to shorten the distance between weak local magnetic moments. However, an excessive content of defects would break the $\pi$-bonding networks and degrade the electric properties of graphene. In analogous $2 \mathrm{D}$ system of graphene like transition metal (TM) dichalcogenides, doping of magnetic $\mathrm{TM}(\mathrm{Fe}, \mathrm{Co}$, and $\mathrm{Ni}$ ) has been experimentally demonstrated to be an effective method for inducing stable roomtemperature ferromagnetic ordering 23,24 . However, direct doping of TM atoms in graphene is impractical because graphene is not an ionic crystal like TM dichalcogenides and its honeycomb networks are maintained by strong $\mathrm{C}-\mathrm{C}$ covalent bonds. This makes the energy barrier of embedding a single TM atom into pristine graphene lattices very steep. Indeed, previous attempts to produce TM-graphene compositions merely yielded TM atoms or clusters adsorbed on the surface of graphene ${ }^{25,26}$, where the magnetism is not intrinsic but mainly contributed by the metal clusters. Meanwhile the adsorbed TM atoms on graphene could be easily dissociated or coagulated at elevated temperatures. For example, graphene with Co atoms attached on the surface can maintain ferromagnetic ordering only below $10 \mathrm{~K}^{26}$. Hence, stable anchoring of magnetic TM atoms on graphene is important for the manufacture of graphene-based room-temperature ferromagnetic materials. Inspired by the metal-nitrogen (M-N) moiety strategies developed recently for atomically dispersed TM catalysts $^{27,28}$, we speculate that doping $\mathrm{TM}$ with the aid of coordinated $\mathrm{N}$ might be a possible way to embed TM atoms into graphene lattices. As the Pauling electronegativity of N (3.0) is significantly higher than that of $\mathrm{C}(2.5)$, the TM atoms can be strongly bound by $\mathrm{N}$ atoms rather than by $\mathrm{C}$ atoms as observed in many TM-N-C systems ${ }^{28,29}$. Therefore, the TM- $\mathrm{N}_{\mathrm{x}}$ moiety can be doped in graphene and act as the ferromagnetic centers. Previous density functional theory (DFT) simulations confirmed the structural stability of the heavy N-doped graphene at up to 1000 $\mathrm{K}^{30}$, further supporting the feasibility of TM- $\mathrm{N}_{\mathrm{x}}$ moiety on building the graphene-based room-temperature ferromagnetic materials.

Herein, motivated by the above consideration, we propose a coordination atom assisted strategy by embedding magnetic TM into graphene lattices under the assistance of coordinated $\mathrm{N}$ atoms, where stable room-temperature ferromagnetism can be achieved in graphene. This strategy is exemplified by the singlemetal-atomic Co- $\mathrm{N}_{4}$ moiety doped graphene, which was synthesized via an impregnation-pyrolysis method, and exhibits unprecedented ferromagnetic orderings with Curie temperature $\left(T_{\mathrm{C}}\right)$ up to $\sim 400 \mathrm{~K}$. A high saturation magnetization of $0.11 \mathrm{emu} \mathrm{g}^{-1}$ at $300 \mathrm{~K}\left(0.73 \mathrm{emu} \mathrm{g}^{-1}\right.$ at $\left.5 \mathrm{~K}\right)$ is obtained at the Co content of 0.4 at. $\%$. Detailed X-ray absorption spectroscopy characterizations and multiple-scattering simulations confirm the embedding of Co atoms in the graphene lattices, via the formation of isolated Co- $\mathrm{N}_{4}$ units. Electronic structure calculations reveal that stable roomtemperature ferromagnetic coupling is mediated by carriers and arises from the $d-p$ orbital hybridization between the embedded $\mathrm{Co}$ and $\mathrm{C} / \mathrm{N}$ atoms. Besides the ferromagnetic ordering, the Co- $\mathrm{N}_{4}$ doped graphene possesses unique electronic structure where only one spin channel crosses the Fermi level, which is beneficial for the generation of spin-polarized current in spintronics ${ }^{31}$. This deliberately designed strategy opens up an avenue for the development of graphene-based spintronic devices.

\section{Results}

Analysis of sample morphology and structure. The atomically dispersed Co embedded N-doped graphene (named 1Co-N/G) was prepared through a two-step synthesis strategy consisting of the impregnation and following pyrolysis procedures. For comparison, samples with other Co contents were prepared, denoted as $0.2 \mathrm{Co}-\mathrm{N} / \mathrm{G}, 0.5 \mathrm{Co}-\mathrm{N} / \mathrm{G}$, and $3 \mathrm{Co}-\mathrm{N} / \mathrm{G}$ with $0.2 \times, 0.5 \times$, and $3 \times$ Co precursors during preparation than that of $1 \mathrm{Co}-\mathrm{N} / \mathrm{G}$ sample (see experimental details in the Methods section). According to the X-ray photoelectron spectroscopy (XPS) analysis (Supplementary Table 1 ), the final Co contents in $0.2 \mathrm{Co}-\mathrm{N} / \mathrm{G}, 0.5 \mathrm{Co}-\mathrm{N} /$ $\mathrm{G}, 1 \mathrm{Co}-\mathrm{N} / \mathrm{G}$, and $3 \mathrm{Co}-\mathrm{N} / \mathrm{G}$ are $0.11,0.22,0.40$, and 1.32 at.\%, respectively. The transmission electron microscopy (TEM) images reveal transparent and corrugation sheets for all the samples (Fig. 1a-c and Supplementary Fig. 1a), typical morphology features for mono- and few-layered graphene-based materials. However, particles with an average diameter of $\sim 20 \mathrm{~nm}$ are observed on the 3Co-N/G nanosheets (Supplementary Fig. 1b), indicating the formation of the second phase; meanwhile no particles were observed in the samples with lower Co contents. We used energy dispersive X-ray (EDX) spectroscopy and aberration corrected high-angle annular dark-field scanning transmission electron microscopy (HAADF-STEM) to further characterize the morphology of $1 \mathrm{Co}-\mathrm{N} / \mathrm{G}$ nanosheets. The EDX images (Fig. 1d) suggest the homogeneous distribution of $\mathrm{C}, \mathrm{N}$, and Co elements over the nanosheets. And in the HAADF-STEM image (Fig. 1e), a number of bright spots (some of them are highlighted by red circles for close observation) in single-atom size, corresponding to the isolated Co atoms, are well dispersed across the nanosheets. We studied the crystalline phase of the 1 Co-N/G nanosheets using X-ray diffraction (XRD), observing a broad peak at about $26.3^{\circ}$ (Supplementary Fig. 2) which can be indexed to the (002) plane of hexagonal graphite ${ }^{32,33}$. No Corelated second phases were detected. Furthermore, Co K-edge Xray absorption near edge structure (XANES) spectra (Fig. If and Supplementary Fig. 3), Fourier-transformed (FT) $k^{3}$-weighted Co K-edge extended X-ray absorption fine structure (EXAFS) spectra (Fig. 1g), and wavelet transform (WT) analysis (Fig. 1h) also 

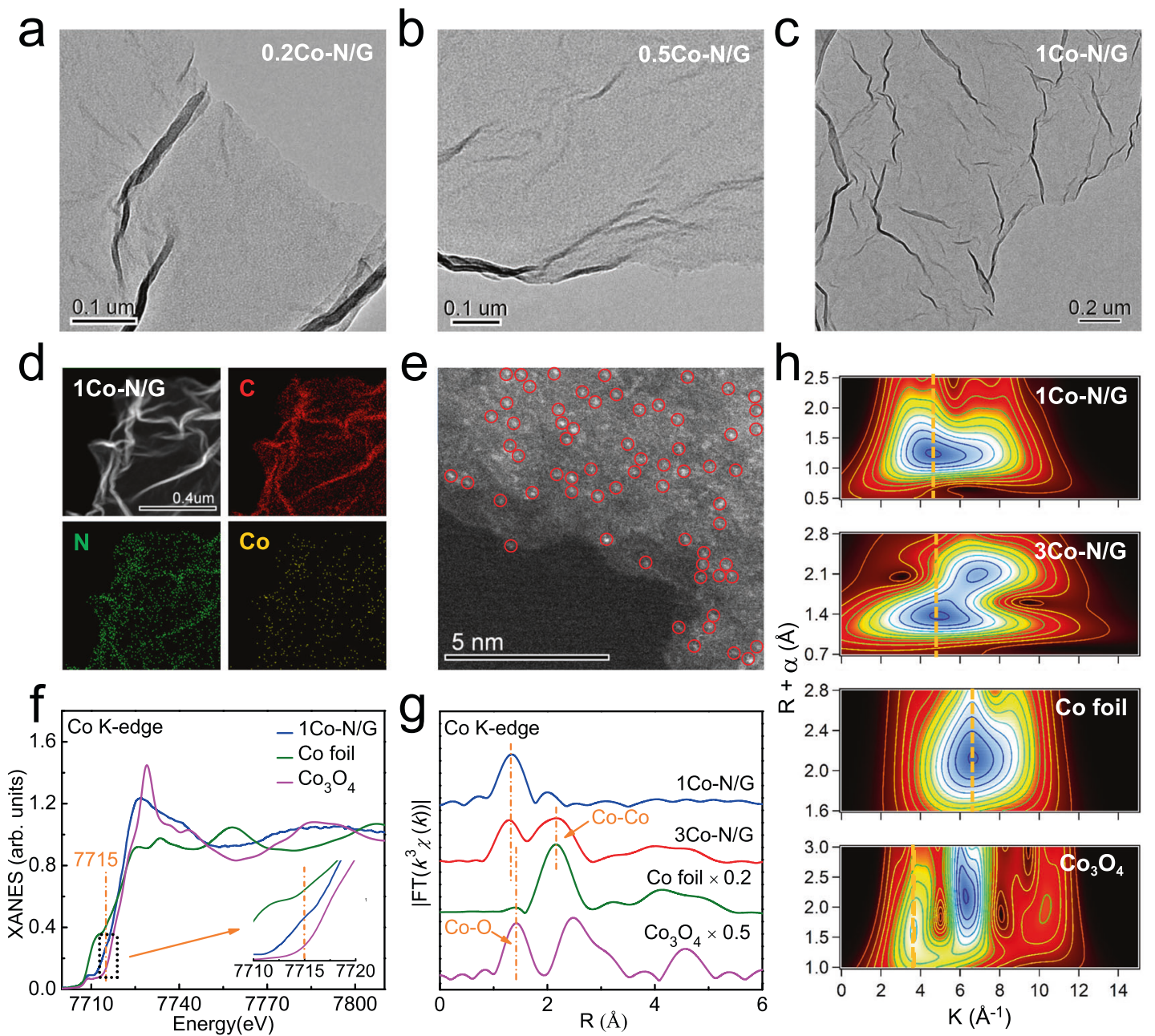

Fig. 1 Atomic dispersion of Co atoms. TEM images of a $0.2 \mathrm{Co}-\mathrm{N} / \mathrm{G}$, b $0.5 \mathrm{Co}-\mathrm{N} / \mathrm{G}$, and $\mathbf{c} 1 \mathrm{Co}-\mathrm{N} / \mathrm{G}$ samples. d EDX elemental mapping and e HAADF-

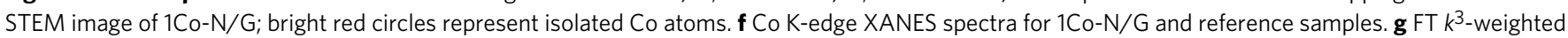
Co K-edge EXAFS spectra and $\mathbf{h}$ WT analysis of EXAFS spectra for 1Co-N/G, 3Co-N/G, and reference samples.

show that Co exists as isolated atoms in graphene (see details in Supplementary Note 1). All the above TEM and XAFS results confirm that atomically dispersed Co in 1Co-N/G nanosheets have been successfully synthesized, without the formation of Corelated second phase when the Co loading is below 0.4 at.\%. When Co content is beyond 0.4 at.\%, part of the Co atoms are in the form of metallic Co nanoparticles (Fig. 1g, h and Supplementary Note 1).

In order to explore the bonding configuration of Co atoms in $1 \mathrm{Co}-\mathrm{N} / \mathrm{G}$ nanosheets, we performed XPS, and N and C K-edge XANES measurements. XPS survey spectrum (Supplementary Fig. 4) clearly shows the existence of $C, N$, and Co elements in $1 \mathrm{Co}-\mathrm{N} / \mathrm{G}$ nanosheets, while no other ferromagnetic impurities were detected. The corresponding fine-scan N $1 s$ XPS spectrum of $1 \mathrm{Co}-\mathrm{N} / \mathrm{G}$ nanosheets shown in Fig. 2a, can be deconvoluted into five component peaks. Besides the $\mathrm{N} 1 s$ signal from of pyridinic $(398.3 \mathrm{eV})$, pyrrolic $(400.0 \mathrm{eV})$, graphitic $(401.2 \mathrm{eV})$, and oxidized $\mathrm{N}$ groups $(402.3 \mathrm{eV})$, there is also a signal from $\mathrm{Co}-\mathrm{N}$ bonds with the XPS peak at $\sim 399.2 \mathrm{eV}^{32,34}$. The formation of Co-N bonds was also confirmed by $\mathrm{N}$ K-edge XANES spectra (Fig. 2b), where an evident absorption peak B derived from Co-N bonding at $\sim 399.5 \mathrm{eV}$ can be observed, which is absent in the N/G nanosheets (see detailed N K-edge XANES analysis in Supplementary Note 2$)^{28,35}$. Further, the C K-edge XANES features of $1 \mathrm{Co}-\mathrm{N} / \mathrm{G}$ nanosheets (Fig. $2 \mathrm{c}$ ) are very similar to those of pristine graphene, indicating similar chemical environments of the carbon matrix, except for a feature at $\sim 287.4 \mathrm{eV}$ corresponding to the $\mathrm{C}$ $\mathrm{N}$ bonding in the $\mathrm{C}$ K-edge XANES of the $1 \mathrm{Co}-\mathrm{N} / \mathrm{G}$ nanosheets (see detailed C K-edge XANES analysis in Supplementary Note 3$)^{36,37}$. Taking these analysis together, we can conclude that there is a specific Co-N-C structural moiety in 1Co-N/G nanosheets. For quantitative analysis on the coordination configuration of $\mathrm{Co}$ atoms in $1 \mathrm{Co}-\mathrm{N} / \mathrm{G}$ nanosheets, a leastsquare EXAFS fitting was performed to extract the atomic structure parameters. Based on bonding configuration analysis, the best-fitting curves in $R$ - and $q$-spaces for $1 \mathrm{Co}-\mathrm{N} / \mathrm{G}$ are shown in Fig. $3 \mathrm{a}$ and Supplementary Fig. 5 by using Co-N backscattering path. The average coordination number of $\mathrm{N}$ atoms is estimated to be 4 with an average scattering distances of $1.95 \AA$ (see details in Supplementary Table 2). It is worthy to note that, as displayed in Fig. 1f, one pronounced pre-edge peak at $\sim 7715 \mathrm{eV}$ can be observed in the Co K-edge XANES spectrum for $1 \mathrm{Co}-\mathrm{N} / \mathrm{G}$ nanosheets. This is a typical feature of the Co- $\mathrm{N}_{4}$ square-planar structure $^{28,38}$, consistent with the EXAFS fitting results indicative of four $\mathrm{N}$ neighbors around a Co atom. The square-planar Co- $\mathrm{N}_{4}$ configuration in $1 \mathrm{Co}-\mathrm{N} / \mathrm{G}$ could be further supported by the Co $\mathrm{L}_{3}$-edge XANES spectrum as shown in Fig. $3 \mathrm{~b}$, exhibits similar fine structure features ( $a, b, c$, and $d$ ) to those of cobaltous phthalocyaninate $(\mathrm{CoPc})$, but significantly different from those of Co metal and oxides as shown in Supplementary Fig. 6. This 

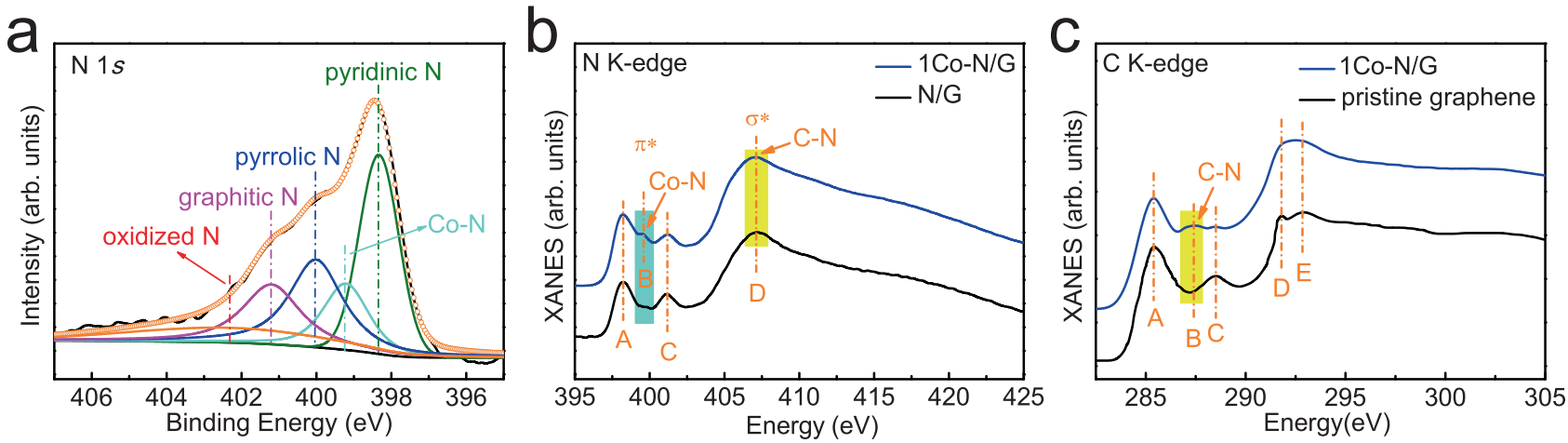

Fig. 2 Bonding configuration of Co atoms. a $N$ 1s XPS spectrum of 1 Co-N/G nanosheets. b N K-edge XANES spectra of 1 Co-N/G and N/G nanosheets. c C K-edge XANES spectra of 1 Co-N/G nanosheets and pristine graphene.
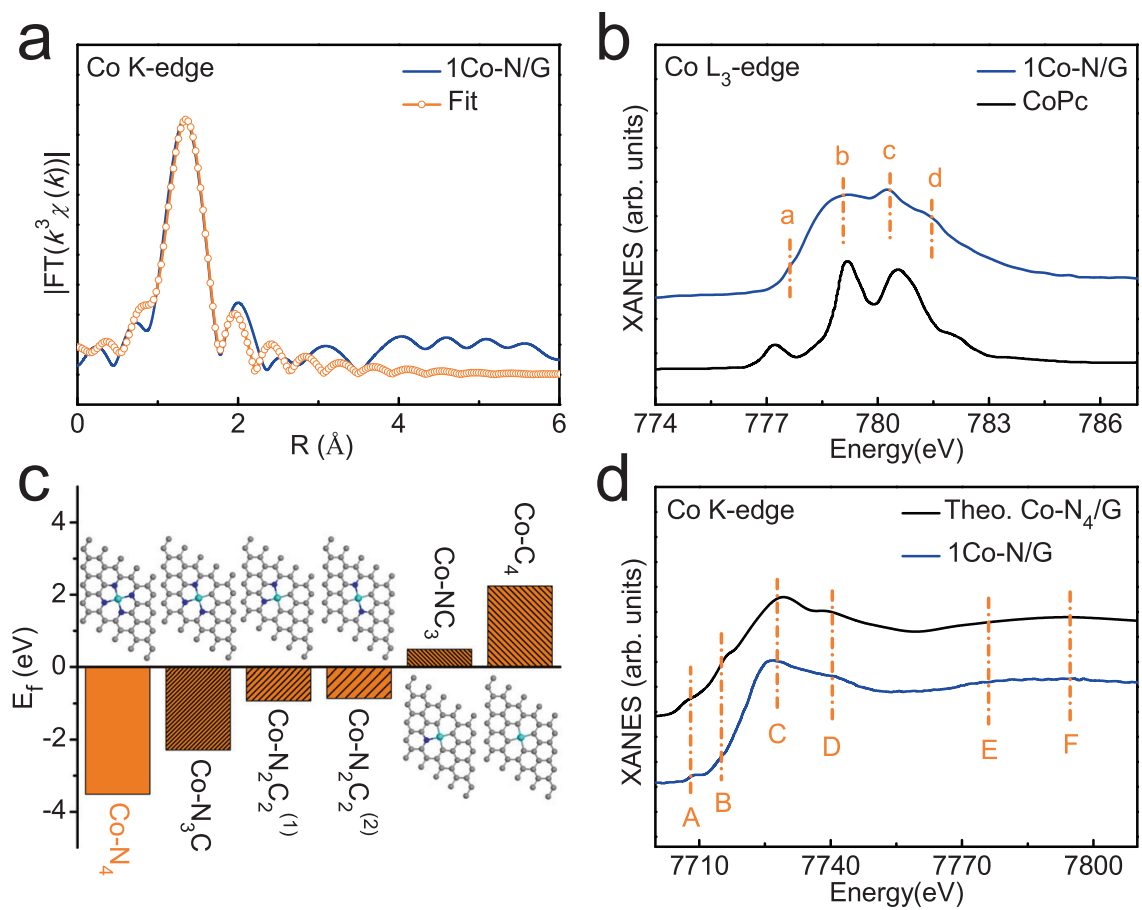

Fig. 3 Coordination configuration around $\mathbf{C o}$ atoms. a Co K-edge EXAFS fitting curves of 1 Co- $N / G$ nanosheets in $R$-space. $\mathbf{b}$ Co $L_{3}$-edge XANES spectra for $1 \mathrm{Co}-\mathrm{N} / \mathrm{G}$ and CoPc reference. c DFT calculated formation energies of various Co- $\mathrm{N}_{4-x} \mathrm{C}_{x}$ moieties in graphene lattices $\left(\mathrm{Co}-\mathrm{N}_{4-x} \mathrm{C}_{x} / \mathrm{G}, X=0,1,2,3\right.$, and 4$)$, together with the structural schematics. The cyan, blue, and black gray spheres represent $\mathrm{Co}, \mathrm{N}$, and $\mathrm{C}$ atoms, respectively. $\mathbf{d}$ Comparison of the experimental Co K-edge XANES spectrum of $1 \mathrm{Co}-\mathrm{N} / \mathrm{G}$ nanosheets and the simulated one based on a model of Co- $\mathrm{N}_{4} / \mathrm{G}$.

indicates that Co atoms are in a similar square-planar crystal field; the difference of crystal field splitting energy (corresponding to the distances between subpeaks) comes from the difference of ligands $s^{39,40}$. Furthermore, considering that the Co-C coordination cannot be unambiguously distinguished from the Co-N coordination by EXAFS analysis, DFT calculations were employed to check the stability of various $\mathrm{CoN}_{4-x} \mathrm{C}_{x}$ moieties in graphene lattices $\left(\mathrm{CoN}_{4-x} \mathrm{C}_{x} / \mathrm{G}, X=0,1,2,3\right.$, and 4) by comparing the formation energies (see calculation details in the Methods section). The calculation results and structural schematics are given in Fig. 3c, where the planar structure of each $\mathrm{CoN}_{4-x} \mathrm{C}_{x} / \mathrm{G}(X=0,1,2,3$, and 4$)$ moiety was ensured to be the converged configuration after structural optimization (see the details in Supplementary Figs. 7 and 8). It is obvious that the Co$\mathrm{N}_{4}$ doped graphene $\left(\mathrm{Co}-\mathrm{N}_{4} / \mathrm{G}\right)$ moiety is much more energetic favorable than other $\mathrm{CoN}_{4-x} \mathrm{C}_{x} / \mathrm{G}(X=1,2,3$, and 4) compounds. In addition, the simulated Co K-edge XANES spectrum based on the structure model of a square-planar
Co- $\mathrm{N}_{4} / \mathrm{G}$ generated by DFT calculations is presented in Fig. 3d, with excellent agreement between the theoretical and experimental XANES spectra. All of the above experimental and theoretical results lead us to conclude that the square-planar Co$\mathrm{N}_{4}$ moieties is the most possible structure for Co atoms in the $1 \mathrm{Co}-\mathrm{N} / \mathrm{G}$ nanosheets.

Ferromagnetism activated by the Co- $\mathrm{N}_{4}$ moieties. Next, to clarify the effect of the Co- $\mathrm{N}_{4}$ moieties on the magnetic properties of graphene nanosheets, we measured the magnetization curves as a function of the applied magnetic field $H(M-H)$ (Fig. 4a) at 300 $\mathrm{K}$ for pristine graphene and $1 \mathrm{Co}-\mathrm{N} / \mathrm{G}$ nanosheets. The welldefined hysteresis loop indicates the room-temperature ferromagnetic behavior of $1 \mathrm{Co}-\mathrm{N} / \mathrm{G}$ nanosheets with saturation magnetization and coercivity of $\sim 0.11 \mathrm{emu} \mathrm{g}^{-1}$ and $142 \mathrm{Oe}$, respectively, different from the non-ferromagnetic properties of pristine graphene as shown in the inset illustration ${ }^{21,22}$. In addition, temperature-dependent magnetization curves $(M-T)$ 

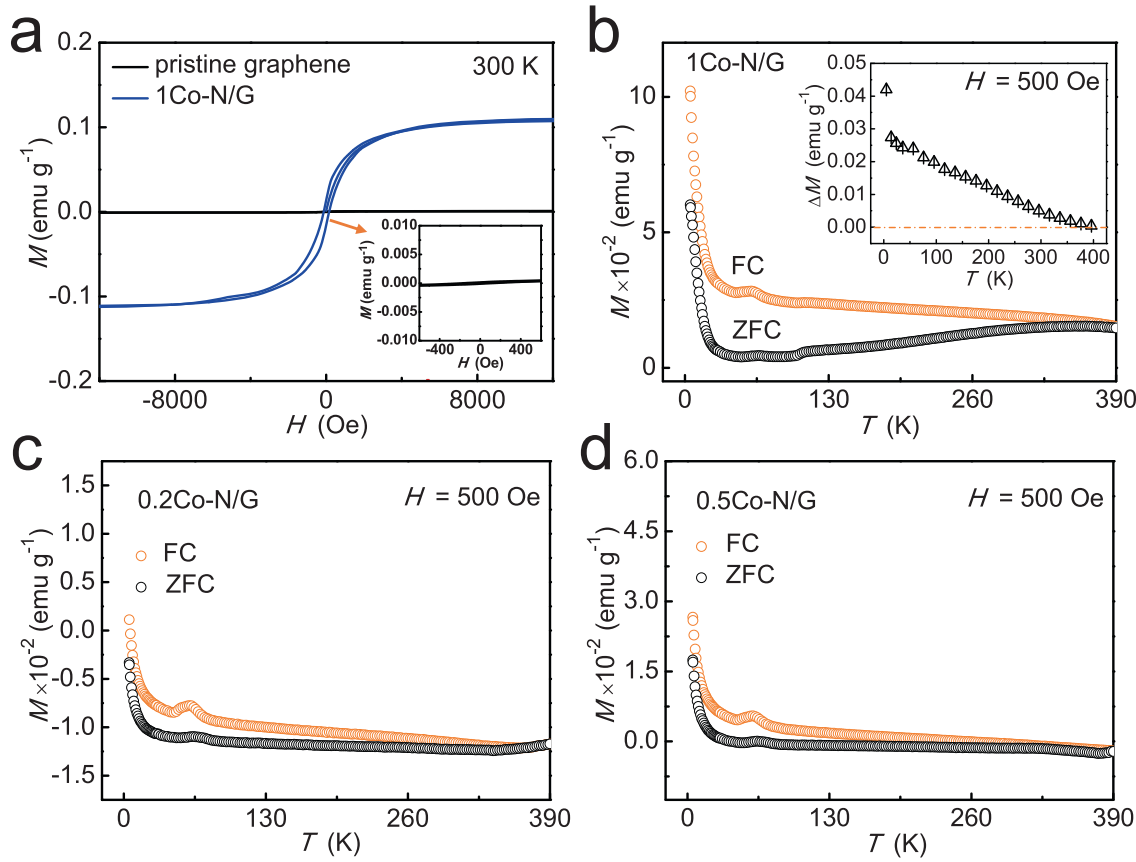

Fig. 4 Magnetic properties. a Magnetization vs. magnetic field $(M-H)$ curves for pristine graphene and 1Co-N/G nanosheets after background subtraction. The inset shows the $M-H$ curves for pristine graphene in narrow scale for better perception. $\mathbf{b}$ Temperature dependence of FC and ZFC magnetization $(M-T)$ curves for $1 C \mathrm{Co}-\mathrm{N} / \mathrm{G}$ nanosheets. Inset: the magnetization difference $(\Delta M)$ between $\mathrm{FC}$ and $\mathrm{ZFC}$ curves $\left(M_{\mathrm{FC}}-M_{\mathrm{ZFC}}\right)$ of 1 Co-N/G nanosheets. c, $\mathbf{d}$ Temperature dependence of FC and ZFC magnetization (M-T) curves for 0.2Co-N/G (c) and 0.5Co-N/G (d) nanosheets.

for $1 \mathrm{Co}-\mathrm{N} / \mathrm{G}$ nanosheets are given in Fig. $4 \mathrm{~b}$ under field-cooling (FC) and zero-field-cooling (ZFC) modes at an applied field of 500 Oe; it is clear that the $1 \mathrm{Co}-\mathrm{N} / \mathrm{G}$ nanosheets have a $T_{\mathrm{C}}$ above room temperature $(300 \mathrm{~K})$. The magnetization difference $(\Delta M)$ between FC and ZFC curves $\left(M_{\mathrm{FC}}-M_{\mathrm{ZFC}}\right)$ in the inset shows a decreasing trend but still perfectly positive in the whole temperature range (up to $\sim 400 \mathrm{~K}$ ). Hence, the possibility of spin glass effects and superparamagnetism can be excluded in the 1Co-N/G nanosheets ${ }^{24,41}$. The corresponding $M-T$ curves for $0.2 \mathrm{Co}-\mathrm{N} / \mathrm{G}$ and $0.5 \mathrm{Co}-\mathrm{N} / \mathrm{G}$ under an applied field of $500 \mathrm{Oe}$ are also depicted in Fig. $4 c$, d, and their $M-H$ curves at $300 \mathrm{~K}$ are given in Supplementary Fig. 9, showing the diamagnetic background contributions in addition to the ferromagnetism, in analogy to previous reports ${ }^{14,17}$. Seen from Fig. $4 \mathrm{~b}-\mathrm{d}$, it is obvious that all the three samples exhibit thermo-magnetic irreversibility (a bifurcation in the FC and ZFC curves) at temperatures above 300 $\mathrm{K}$. And the cusp at about $65 \mathrm{~K}$ can be attributed to molecular oxygen on the surface ${ }^{42-45}$. Furthermore, in order to visually show the change of magnetism with Co contents in doped graphene nanosheets, we extracted magnetic signals from the $M-H$ curves for all the samples and plotted them against the Co contents in Supplementary Fig. 10. Obviously, the saturation magnetization for as-synthesized samples is enhanced when the Co content increases from $0.2 \times$ to $1.0 \times \mathrm{Co}$. At the content of $3.0 \times$ $\mathrm{Co}$, according to the above characterization analysis, the ferromagnetism is not intrinsic and partly comes from the contribution of Co-based nanoparicles or/and clusters. At the temperature of $5 \mathrm{~K}$, the $M-H$ curves for $1 \mathrm{Co}-\mathrm{N} / \mathrm{G}$ nanosheets exhibit similar ferromagnetism with higher saturation magnetization of $\sim 0.73$ $\mathrm{emu} \mathrm{g}^{-1}$ (Supplementary Fig. 13). If there is ferromagnetism derived from Co metal clusters, by comparing the magnetic moment of $1 \mathrm{Co}-\mathrm{N} / \mathrm{G}$ samples measured at $5 \mathrm{~K}\left(\sim 0.4 \mu_{\mathrm{B}} \mathrm{Co}^{-1}\right)$ with that of Co metal $\left(1.7 \mu_{\mathrm{B}} \mathrm{Co}^{-1}\right)$, we can estimate that $\sim 24 \%$ of the total doped Co atoms should be in the form of clusters. Such a content of Co clusters should be easily detected by TEM and XANES $^{46}$, which is however contrary to the experimental observations (Fig. 1c, f). Meanwhile, the $T_{\mathrm{C}}$ of $1 \mathrm{Co}-\mathrm{N} / \mathrm{G}$ at about $400 \mathrm{~K}$ is much lower than that of Co metal clusters. In addition, we treated intrinsic non-magnetic $\mathrm{Al}_{2} \mathrm{O}_{3}$ powder under the same experimental and magnetic characterization conditions as for the 1 Co-N/G nanosheets. No ferromagnetism was observed, which helps ruling out the possibility of unintentional introduction of magnetic impurities during the experimental process. We can conclude that the ferromagnetism in the Co- $\mathrm{N}_{4}$ moiety doped graphene nanosheets is intrinsic.

Origin of the ferromagnetism in $\mathrm{CoN}_{4}$-graphene system. To obtain an in-depth understanding on the origin of the roomtemperature ferromagnetic coupling in $\mathrm{CoN}_{4}$-graphene system, we performed spin-polarize DFT calculations (see calculation details in the section of Methods). The total densities of states (TDOS), projected densities of states (PDOS), energy band structure and spatial distribution of spin density are presented in Fig. 5a-e, respectively. For reference, Fig. 5a shows the TDOS of pristine graphene, which has no spin polarization and is semimetallic with Dirac cone at the Fermi level (corresponding to $E=$ $0 \mathrm{eV}$ in the DOS $)^{47,48}$. In contrast, the TDOS and PDOS of $\mathrm{CoN}_{4-}$ graphene system (Fig. 5b, c) exhibits a significantly enhanced and spin-polarized DOS around the Fermi level. According to the Stoner criterion ${ }^{49,50}$, a high DOS at the Fermi level ensures ferromagnetism at relatively high temperature. In order to show the band structure of $\mathrm{CoN}_{4}$-graphene more clearly, we made a stack diagram of DFT calculated total and projected DOSs for $\mathrm{CoN}_{4-}$ graphene system (Supplementary Fig. 14). It is obvious that the system exhibits a metallic character with non-zero DOS at the Fermi level. In addition, we calculated the $2 \mathrm{D}$ plot of the energy band structure for $\mathrm{CoN}_{4}$-graphene as shown in Fig. $5 \mathrm{~d}$, where the Fermi level crosses some energy bands and no significant bandgap can be observed, consistent with the DOS (Fig. 5b, c and Supplementary Fig. 14) and previous theoretical calculations ${ }^{51}$. Further, the PDOS in Fig. $5 \mathrm{c}$ reveals the intense hybridization 

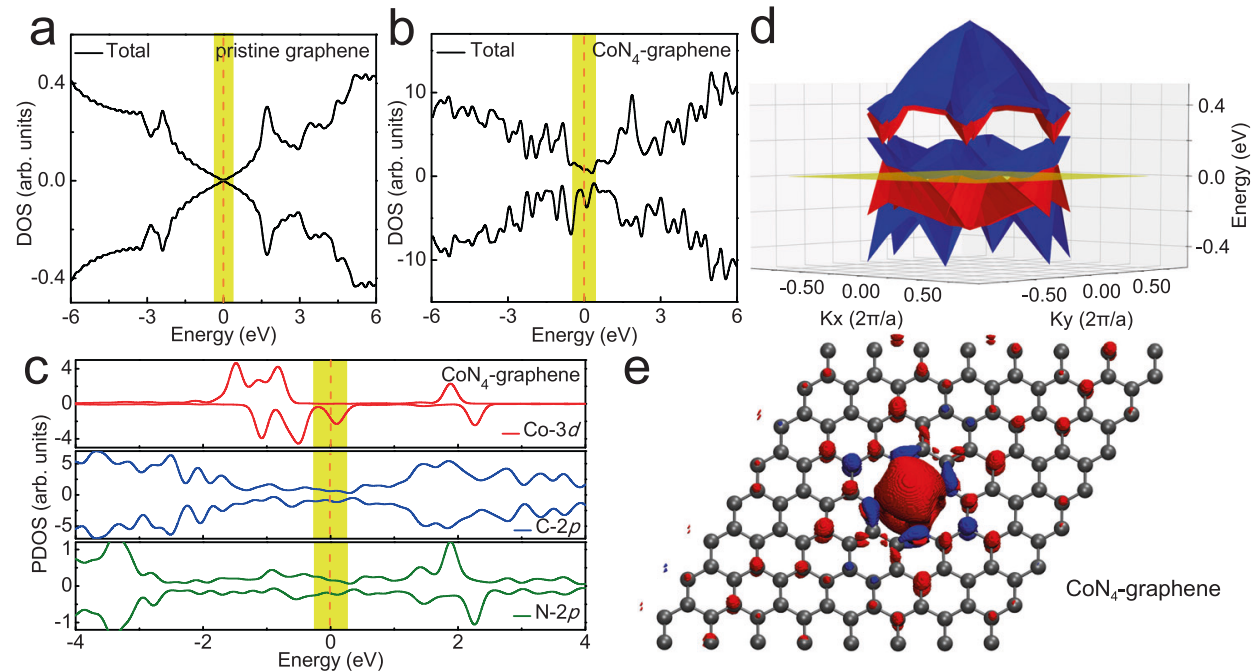

Fig. 5 Electronic structure and origin of ferromagnetism. DFT calculated TDOS for a pristine graphene and $\mathbf{b}$ CoN $\mathrm{N}_{4}$-graphene system. $\mathbf{c}$ DFT calculated PDOS for $\mathrm{CoN}_{4}$-graphene system. $\mathbf{d} 2 \mathrm{D}$ plot of the energy band structure for $\mathrm{CoN}_{4}$-graphene. The red and blue surfaces represent the bands with up and down spin directions, respectively. The transparent yellow plain corresponds to the Fermi level $(E=0 \mathrm{eV})$. e Spatial distribution of spin density ( $\rho \uparrow-\rho \downarrow)$ for a $8 \times 8 \times 1$ hexagonal graphene supercell with one doped square-planar $\mathrm{Co}_{0} \mathrm{~N}_{4}$ structure. Red and blue isosurfaces represent positive and negative spin densities, respectively.

between the Co- $3 d, \mathrm{~N}-2 p$, and C-2p electronic bands. The spatial distribution of spin density (Fig. $5 \mathrm{e}$ ) also indicates that the spinpolarized electrons in the graphene matrix are mainly delocalized $p_{\mathrm{z}}$ electrons. Bipartite arrangements of spin polarization can also be observed in the spatial distribution of spin density; the $\mathrm{N}$ atoms dictate the arrangement of spin polarizations in its own sublattices. Hence the magnetic states between two Co atoms should depend on their relative positions ${ }^{52}$, which is proved by the calculated relative energies of ferromagnetic (FM) and antiferromagnetic (AFM) states of different bi-Co- $\mathrm{N}_{4}$ configurations (Supplementary Fig. 15). The coexistence of FM and AFM states agrees with the fact that the measured magnetic moment is smaller than the predicted value $\left(1.0 \mu_{\mathrm{B}} / \mathrm{Co}\right)^{51,53}$. Due to the intense hybridization between Co $d$ and delocalized N/C $p_{z}$ orbits, the long-range magnetic coupling between Co atoms can be attributed to an indirect conduction-electron mediated Ruderman-Kittel-Kasuya-Yosida (RKKY)-like interaction ${ }^{54,55}$. Meanwhile, we have also carefully considered other magnetic coupling frameworks including successive spin polarization-based ferromagnetic coupling (SSP-FMC) 56,57 and bound magnetic polaron (BMP) ${ }^{58}$ that have successfully interpreted the origins of ferromagnetism in many TM-doped system. However, due to the metallic nature, enhanced DOS at Fermi level and spin-polarized $\pi$ electrons for our $\mathrm{CoN}_{4}$-graphene system, the conductionelectron mediated ferromagnetism is a more reasonable and straightforward explanation for the main origin of ferromagnetism in $\mathrm{CoN}_{4}$-graphene nanosheets. Since the hybridization between the Co-3d, N-2p, and C-2p electronic bands is essential for the development of the ferromagnetic coupling in $\mathrm{CoN}_{4}$ graphene nanosheets, the strong room-temperature ferromagnetism originates from the chemical bonding that embeds Co atoms into the graphene lattice. Hence the DFT simulations lead us to conclude that the $\mathrm{Co}-\mathrm{N}_{4}$ moieties are responsible for the observed room-temperature ferromagnetism in the assynthesized $\mathrm{CoN}_{4}$-graphene nanosheets.

Finally, we summarize the important role of co-doped $\mathrm{N}$ atoms in the establishment of graphene magnetism: (1) $\mathrm{N}$ atoms serve as a stable anchoring site for Co due to the higher Pauling electronegativity than that of $\mathrm{C}$ atoms, which avoids the loss of ferromagnetic Co atoms from graphene lattice. (2) Compared with $\mathrm{C}$ atoms, $\mathrm{N}$ possess one additional $p$ electron, making them an n-type dopant in graphene, which can effectively improve the electric conductivity of graphene and enhance the DOS around the Fermi level to meet the Stoner criterion. (3) In the context of the bipartite nature of graphene, the $\mathrm{N}$ atoms induce a bipartite arrangement of magnetic moments of the sublattices where they are. Moreover, it is worth noting that the $\mathrm{CoN}_{4}$-graphene nanosheets have greatly improved performance of spinpolarized current because only one spin channel crosses the Fermi level ${ }^{31}$.

\section{Discussion}

In summary, we have explored the possibility of achieving stable room-temperature ferromagnetic ordering in graphene by embedding single magnetic TM atoms in the lattice via the strong chemical bonds in the TM- $\mathrm{N}_{\mathrm{x}}$ moieties. A simple and controllable impregnation-pyrolysis process was employed to synthesize the $\mathrm{Co}-\mathrm{N}$-graphene system. Comprehensive structural characterizations confirm the existence of square-planar Co- $\mathrm{N}_{4}$ moieties in the graphene lattices at the content of 0.4 at.\% (for $1 \mathrm{Co}-\mathrm{N} / \mathrm{G}$ nanosheets), which displays unprecedented stable ferromagnetic ordering with $T_{\mathrm{C}}$ up to $\sim 400 \mathrm{~K}$ and saturation magnetization of $0.11 \mathrm{emu} \mathrm{g}^{-1}(300 \mathrm{~K})$. No Co-related second phase that may induce extrinsic ferromagnetism is observed. On the one hand, Co- $\mathrm{N}_{4}$ moieties avoid the loss of Co atoms from the graphene lattices, thus providing a strong local magnetic moment; on the other hand, DFT calculation reveals that the long-range ferromagnetic coupling originates from the hybridization between the Co- $3 d$ and $\mathrm{C} / \mathrm{N}-2 p$ orbitals through a carrier-mediated magnetic interaction. The stable room-temperature graphene magnets developed in this work will pave the way towards practical graphene-based spintronic applications.

\section{Methods}

Synthesis of 1Co-N/G and N/G nanosheets. 1Co-N/G nanosheets were synthesized through a two-step impregnation-pyrolysis procedure. In the typical procedure, graphene oxide (GO) was firstly prepared by oxidation of powdered flake graphite following a modified Hummers' method ${ }^{59}$. Then, $50 \mu \mathrm{l}$ of $\mathrm{CoCl}_{2} \cdot 6 \mathrm{H}_{2} \mathrm{O}$ solution $\left(3 \mathrm{mg} \mathrm{ml}^{-1}\right)$ and $15 \mu \mathrm{l}$ of $\mathrm{H}_{2} \mathrm{O}_{2}$ solution (30\%) were added dropwise into $10 \mathrm{ml}$ of GO suspension $\left(2 \mathrm{mg} \mathrm{ml}^{-1}\right)$ and the mixture was sonicated for $1 \mathrm{~h}$. The obtained suspension was transferred into a stainless steel autoclave and maintained at $180^{\circ} \mathrm{C}$ for $6 \mathrm{~h}$, forming a hydrogel. After freeze drying, the $\mathrm{Co}^{2+}$. containing gel was heated in the center of a quartz tube furnace at $900{ }^{\circ} \mathrm{C}$ for $1 \mathrm{~h}$ 
under gas flows of $100 \mathrm{sccm}$ Ar and $50 \mathrm{sccm} \mathrm{NH}_{3}$ to obtain 1Co-N/G. For comparison, the N/G nanosheets were prepared with the same procedure with no addition of $\mathrm{CoCl}_{2} \cdot 6 \mathrm{H}_{2} \mathrm{O}$.

Synthesis of $\mathbf{0 . 2 C o}-\mathbf{N} / \mathbf{G}, \mathbf{0 . 5} \mathrm{Co}-\mathbf{N} / \mathbf{G}$, and $\mathbf{3 C o}-\mathbf{N} / \mathbf{G}$ nanosheets. $0.2 \mathrm{Co}-\mathrm{N} / \mathrm{G}$, $0.5 \mathrm{Co}-\mathrm{N} / \mathrm{G}$, and $3 \mathrm{Co}-\mathrm{N} / \mathrm{G}$ nanosheets were synthesized with a similar procedure to $1 \mathrm{Co}-\mathrm{N} / \mathrm{G}$ except for $0.2 \times, 0.5 \times$, and $3 \times$ Co precursors during preparation, respectively.

DFT calculation details. The spin-polarized DFT calculations were performed with Quantum Espresso software package ${ }^{60}$ and some calculation results were visualized with VMD software ${ }^{61}$. The electron exchange-correlation was processed within the framework of generalized gradient approximation (GGA) in the Perdew-Burke-Ernzerhof (PBE) parametrization. The electron-ion interaction was described using the projected augmented wave (PAW) method. The kinetic energy cutoffs of the plane wave and electron density were 80 and $500 \mathrm{Ry}$. The long-range van der Waals interaction was considered with the DFT-D3 scheme ${ }^{62}$. A $6 \times 6 \times 1$ k-grid was used for sampling in the Brillouin zone. Energy convergence of $\sim 1.0 \times$ $10^{-3} \mathrm{meV} /$ atom was ensured during the self-consistent field calculations. And the convergence criteria for the atomic forces was $0.05 \mathrm{eV} / \mathrm{A}$. Various typical planar and nonplanar configurations have been fully considered during the structure optimizations, as the examples shown in Supplementary Figs. 7 and 8

The Co embedded nitrogen-doped graphene nanosheets were modeled based on an $8 \times 8 \times 1$ hexagonal supercell of graphene. Six adjacent carbon atoms were replaced with $\mathrm{Co}-\mathrm{N}_{4}$. A vacuum layer of $15 \AA$ was placed along the $\mathrm{z}$ direction to prevent the interlayer interaction.

The formation energies of Co- $\mathrm{N}_{x} \mathrm{C}_{4-x}$ moieties in graphene were calculated according to the following equation,

$$
E_{f}=E_{\left[\mathrm{Co}-\mathrm{N}_{x} \mathrm{C}_{4-x}\right]}+(2+x) \mu_{\mathrm{C}}-64 \mu_{\mathrm{C}}-x \mu_{\mathrm{N}}-E_{[\mathrm{Co}(\mathrm{g})]}
$$

where $E_{\left[\mathrm{Co}-\mathrm{N}_{x} \mathrm{C}_{4-x}\right]}$ is the total energy of an $8 \times 8 \times 1$ graphene supercell with a Co$\mathrm{N}_{x} \mathrm{C}_{4-x}$ moiety; $\mu_{\mathrm{C}}$ and $\mu_{\mathrm{N}}$ are the chemical potentials of $\mathrm{C}$ and $\mathrm{N}$, defined as the total energy per atom in graphene and nitrogen molecules; $\mathrm{E}_{[\mathrm{Co}(\mathrm{g})]}$ is the total energy of an isolated Co atoms.

Characterization. TEM and EDX spectroscopy results were taken on a field emission transmission electron microscope (JEM-2100F) with $200 \mathrm{KV}$ accelerating voltage. The HAADF-STEM images were captured on a JEM-ARM200F instrument at $200 \mathrm{KV}$. The XRD and XPS patterns were collected on a Philips X'Pert Pro Super diffractometer with $\mathrm{Cu} \mathrm{Ka}$ line $(\lambda=1.54178 \AA)$ and an ESCALAB MKII equipped with $\mathrm{Mg} K a$ source $(\mathrm{h} v=1253.6 \mathrm{eV})$, respectively. The N K-edge, C Kedge, and $\mathrm{Mo} \mathrm{L}_{3}$-edge XANES were obtained at the BL12B beamline of National Synchrotron Radiation Laboratory (NSRL, China) under a total electron yield (TEY) mode with vacuum better than $5 \times 10^{-7} \mathrm{~Pa}$. The Co K-edge EXAFS spectra were obtained on the 1W1B beamline of Beijing Synchrotron Radiation Facility (BSRF, China) under fluorescence mode. The temperature- and magnetic fielddependent magnetization curves were measured on a superconducting quantum interference device (SQUID) magnetometer.

\section{Data availability}

The data that support the findings of this study are available from the corresponding author upon reasonable request.

Received: 20 July 2020; Accepted: 25 February 2021;

Published online: 25 March 2021

\section{References}

1. Li, D. \& Kaner, R. B. Graphene-based materials. Science 320, 1170-1171 (2008).

2. Stankovich, S. et al. Graphene-based composite materials. Nature 442, 282-286 (2006).

3. Stoller, M. D., Park, S., Zhu, Y., An, J. \& Ruoff, R. S. Graphene-based ultracapacitors. Nano Lett. 8, 3498-3502 (2008).

4. Lee, C., Wei, X., Kysar, J. W. \& Hone, J. Measurement of the elastic properties and intrinsic strength of monolayer graphene. Science 321, 385-388 (2008).

5. Novoselov, K. S. et al. Two-dimensional gas of massless dirac fermions in graphene. Nature 438, 197-200 (2005).

6. Banszerus, L. et al. Ultrahigh-mobility graphene devices from chemical vapor deposition on reusable copper. Sci. Adv. 1, e1500222 (2015).

7. Balandin, A. A. et al. Superior thermal conductivity of single-layer graphene. Nano Lett. 8, 902-907 (2008).

8. Vicarelli, L. et al. Graphene field-effect transistors as room-temperature terahertz detectors. Nat. Mater. 11, 865-871 (2012).
9. Liu, Y., Dong, X. \& Chen, P. Biological and chemical sensors based on graphene materials. Chem. Soc. Rev. 41, 2283-2307 (2012).

10. Antolini, E. Graphene as a new carbon support for low-temperature fuel cell catalysts. Appl. Catal. B Environ. 123, 52-68 (2012).

11. Tombros, N. et al. Electronic spin transport and spin precession in single graphene layers at room temperature. Nature 448, 571-574 (2007).

12. Georgakilas, V., Perman, J. A., Tucek, J. \& Zboril, R. Broad family of carbon nanoallotropes: classification, chemistry, and applications of fullerenes, carbon dots, nanotubes, graphene, nanodiamonds, and combined superstructures. Chem. Rev. 115, 4744-4822 (2015).

13. Sepioni, M. et al. Limits on intrinsic magnetism in graphene. Phys. Rev. Lett. 105, 207205 (2010).

14. Tucek, J. et al. Sulfur doping induces strong ferromagnetic ordering in graphene: effect of concentration and substitution mechanism. Adv. Mater. 28, 5045-5053 (2016).

15. Palacios, J. J., Fernández-Rossier, J. \& Brey, L. Vacancy-induced magnetism in graphene and graphene ribbons. Phys. Rev. B 77, 195428 (2008).

16. Tucek, J. et al. Room temperature organic magnets derived from $s p^{3}$ functionalized graphene. Nat. Commun. 8, 14525 (2017).

17. Blonski, P. et al. Doping with graphitic nitrogen triggers ferromagnetism in graphene. J. Am. Chem. Soc. 139, 3171-3180 (2017).

18. González-Herrero, H. et al. Atomic-scale control of graphene magnetism by using hydrogen atoms. Science 352, 437-441 (2016).

19. Magda, G. Z. et al. Room-temperature magnetic order on zigzag edges of narrow graphene nanoribbons. Nature 514, 608-611 (2014).

20. Ito, Y. et al. Tuning the magnetic properties of carbon by nitrogen doping of its graphene domains. J. Am. Chem. Soc. 137, 7678-7685 (2015).

21. Nair, R. R. et al. Spin-half paramagnetism in graphene induced by point defects. Nat. Phys. 8, 199-202 (2012).

22. Liu, Y. et al. Elemental superdoping of graphene and carbon nanotubes. Nat. Commun. 7, 10921 (2016)

23. Ahmed, S. et al. Magnetic properties of Co doped WSe $\mathrm{W}_{2}$ by implantation. J. Alloy. Compd 731, 25-31 (2018).

24. Duan, H. et al. Beating the exclusion rule against the coexistence of robust luminescence and ferromagnetism in chalcogenide monolayers. Nat. Commun. 10, 1584 (2019).

25. Matsui, D. et al. Magnetic properties of cobalt-carbon nanocomposites. Phys. Status Solidi C. 7, 1264-1268 (2010).

26. Hota, P., Akhtar, A., Bhattacharya, S., Miah, M. \& Saha, S. K. Ferromagnetism in graphene due to charge transfer from atomic Co to graphene. Appl. Phys. Lett. 111, 042402 (2017).

27. Wan, X. et al. Fe-N-C electrocatalyst with dense active sites and efficient mass transport for high-performance proton exchange membrane fuel cells. Nat. Catal. 2, 259-268 (2019).

28. Du, Z. et al. Cobalt in nitrogen-doped graphene as single-atom catalyst for high-sulfur content lithium-sulfur batteries. J. Am. Chem. Soc. 141, 3977-3985 (2019)

29. Sun, T. et al. Single-atomic cobalt sites embedded in hierarchically ordered porous nitrogen-doped carbon as a superior bifunctional electrocatalyst. Proc. Natl Acad. Sci. USA 115, 12692-12697 (2018).

30. Chaban, V. V. \& Prezhdo, O. V. Nitrogen-nitrogen bonds undermine stability of N-doped graphene. J. Am. Chem. Soc. 137, 11688-11694 (2015).

31. Sun, Y. L. et al. Itinerant ferromagnetic half metallic cobalt-iron couples: promising bifunctional electrocatalysts for ORR and OER. J. Mater. Chem. A 7, 27175-27185 (2019).

32. Zhu, Y. et al. One-pot pyrolysis to $\mathrm{N}$-doped graphene with high-density Pt single atomic sites as heterogeneous catalyst for alkene hydrosilylation. ACS Catal. 8, 10004-10011 (2018).

33. Yang, Z. et al. Boosting oxygen reduction catalysis with $\mathrm{Fe}-\mathrm{N}_{4}$ sites decorated porous carbons toward fuel cells. ACS Catal. 9, 2158-2163 (2019).

34. Zhu, C. et al. Hierarchically porous $\mathrm{M}-\mathrm{N}-\mathrm{C}(\mathrm{M}=\mathrm{Co}$ and $\mathrm{Fe})$ single-atom electrocatalysts with robust $\mathrm{MN}_{\mathrm{x}}$ active moieties enable enhanced ORR performance. Adv. Energy Mater. 8, 1801956 (2018).

35. Zhao, L. et al. Cascade anchoring strategy for general mass production of high-loading single-atomic metal-nitrogen catalysts. Nat. Commun. 10, 1278 (2019).

36. Fei, H. et al. Microwave-assisted rapid synthesis of graphene-supported single atomic metals. Adv. Mater. 30, 1802146 (2018).

37. Zheng, Y. et al. Hydrogen evolution by a metal-free electrocatalyst. Nat. Commun. 5, 3783 (2014).

38. Alves, M. M., Dodelet, J., Guay, D., Ladouceur, M. \& Tourillon, G. Origin of the electrocatalytic properties for oxygen reduction of some heat-treated polyacrylonitrile and phthalocyanine cobalt compounds adsorbed on carbon black as probed by electrochemistry and X-ray absorption spectroscopy. J. Phys. Chem. 96, 10898-10905 (1992).

39. Zhang, T. et al. Conclusively addressing the CoPc electronic structure: A joint gas-phase and solid-state photoemission and absorption spectroscopy study. J. Phys. Chem. C 121, 26372-26378 (2017). 
40. Willey, T. M. et al. Electronic structure differences between $\mathrm{H}_{2}-$, Fe-, Co-, and $\mathrm{Cu}$-phthalocyanine highly oriented thin films observed using NEXAFS spectroscopy. J. Chem. Phys. 139, 034701 (2013).

41. Hebard, A. et al. Mining for high Tc ferromagnetism in ion-implanted dilute magnetic semiconductors. J. Phys. D Appl. Phys. 37, 511-517 (2004).

42. Felner, I. Peculiar magnetic features and superconductivity in sulfur doped amorphous carbon. Magnetochemistry 2, 34 (2016).

43. Bandow, S., Yamaguchi, T. \& Iijima, S. Magnetism of adsorbed oxygen on carbon nanohorns. Chem. Phys. Lett. 401, 380-384 (2005).

44. Ben Dor, O., Yochelis, S., Mathew, S. P., Naaman, R. \& Paltiel, Y. A chiralbased magnetic memory device without a permanent magnet. Nat. Commun. 4, 2256 (2013)

45. Felner, I. \& Prilutskiy, E. Unusual magnetic properties and superconductivity in sulfur-doped amorphous carbon powder. J. Supercond. Nov. Magn. 25, 2547-2555 (2012).

46. Griffin, K. A., Pakhomov, A. B., Wang, C. M., Heald, S. M. \& Krishnan, K. M. Intrinsic ferromagnetism in insulating cobalt doped anatase $\mathrm{TiO}_{2}$. Phys. Rev. Lett. 94, 157204 (2005).

47. Zheng, J., Ren, Z., Guo, P., Fang, L. \& Fan, J. Diffusion of $\mathrm{Li}^{+}$ion on graphene: A DFT study. Appl. Surf. Sci. 258, 1651-1655 (2011).

48. Majidi, R. \& Karami, A. Adsorption of formaldehyde on graphene and graphyne. Phys. E 59, 169-173 (2014).

49. Stoner, E. C. Collective electron ferromagnetism. Proc. R. Soc. Lond. 165 0372-0414 (1938).

50. Al Ma'Mari, F. et al. Beating the Stoner criterion using molecular interfaces. Nature 524, 69-U128 (2015).

51. Kattel, S., Atanassov, P. \& Kiefer, B. Stability, electronic and magnetic properties of in-plane defects in graphene: a first-principles study. J. Phys. Chem. C 116, 8161-8166 (2012).

52. Lisenkov, S., Andriotis, A. N. \& Menon, M. Magnetic anisotropy and engineering of magnetic behavior of the edges in Co embedded graphene nanoribbons. Phys. Rev. Lett. 108, 187208 (2012).

53. Krasheninnikov, A. V., Lehtinen, P. O., Foster, A. S., Pyykko, P. \& Nieminen, R. M. Embedding transition-metal atoms in graphene: structure, bonding, and magnetism. Phys. Rev. Lett. 102, 126807 (2009).

54. Santos, E. J. G., Sanchez-Portal, D. \& Ayuela, A. Magnetism of substitutional Co impurities in graphene: Realization of single pi vacancies. Phys. Rev. B 81, 125433 (2010).

55. Park, H., Wadehra, A., Wilkins, J. W. \& Castro Neto, A. H. Spin-polarized electronic current induced by sublattice engineering of graphene sheets with boron/nitrogen. Phys. Rev. B 87, 085441 (2013).

56. Andriotis, A. N. \& Menon, M. Defect-induced magnetism: codoping and a prescription for enhanced magnetism. Phys. Rev. B 87, 155309 (2013).

57. Andriotis, A. N., Fthenakis, Z. G. \& Menon, M. Successive spin polarizations underlying a new magnetic coupling contribution in diluted magnetic semiconductors. J. Phys. Condens. Matter 27, 052202 (2015).

58. Coey, J., Venkatesan, M. \& Fitzgerald, C. Donor impurity band exchange in dilute ferromagnetic oxides. Nat. Mater. 4, 173 (2005).

59. Hummers, W. S. Jr \& Offeman, R. E. Preparation of graphitic oxide. J. Am Chem. Soc. 80, 1339-1339 (1958).

60. Giannozzi, P. et al. QUANTUM ESPRESSO: a modular and open-source software project for quantum simulations of materials. J. Phys. Condens. Matter 21, 395502 (2009).

61. Humphrey, W., Dalke, A. \& Schulten, K. VMD: visual molecular dynamics. J. Mol. Graph. 14, 33-38 (1996).
62. Grimme, S., Ehrlich, S. \& Goerigk, L. Effect of the damping function in dispersion corrected density functional theory. J. Comput. Chem. 32, 1456-1465 (2011)

\section{Acknowledgements}

This work was financially supported by the National Natural Science Foundation of China (Grants No.11975234, 11775225, 12075243, and 12005227), the Users with Excellence Program of Hefei Science Center CAS (No. 2019HSC-UE002, 2020HSCUE002, and 2020HSC-CIP013), the Postdoctoral Science Foundation of China (Grants No. 2019M662202, 2020M682041, and 2020TQ0316), and partially carried out at the USTC Center for Micro and Nanoscale Research and Fabrication. The authors would like to thank BSRF, SSRF, and NSRL for the synchrotron radiation beamtime.

\section{Author contributions}

C.W., Z.S., and W.Y. conceived the experiments and supervised the project; W.H., H.T., H.D., and F.H. performed the measurements; W.H., H.L., N.L., and Q.J. performed the magnetism measurements; C.W. performed the DFT calculations; W.H., C.W., Y.L., and Y.W. analyzed the results; and W.H., C.W., Z.S., and W.Y. wrote the paper with comments from all authors.

\section{Competing interests}

The authors declare no competing interests.

\section{Additional information}

Supplementary information The online version contains supplementary material available at https://doi.org/10.1038/s41467-021-22122-2.

Correspondence and requests for materials should be addressed to C.W., Z.S. or W.Y.

Peer review information Nature Communications thanks Antonis N Andriotis and Jiabao Yi for their contribution to the peer review of this work.

Reprints and permission information is available at http://www.nature.com/reprints

Publisher's note Springer Nature remains neutral with regard to jurisdictional claims in published maps and institutional affiliations.

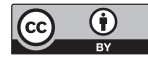

Open Access This article is licensed under a Creative Commons Attribution 4.0 International License, which permits use, sharing, adaptation, distribution and reproduction in any medium or format, as long as you give appropriate credit to the original author(s) and the source, provide a link to the Creative Commons license, and indicate if changes were made. The images or other third party material in this article are included in the article's Creative Commons license, unless indicated otherwise in a credit line to the material. If material is not included in the article's Creative Commons license and your intended use is not permitted by statutory regulation or exceeds the permitted use, you will need to obtain permission directly from the copyright holder. To view a copy of this license, visit http://creativecommons.org/ licenses/by/4.0/.

(C) The Author(s) 2021 